\title{
Pheochromocytoma Invaded by Metastatic Lung Carcinoma
}

\author{
${ }^{1}$ Radu Mihai, ${ }^{2}$ Derek E Roskell, ${ }^{3}$ Chandi Ratnatunga, ${ }^{4}$ Gregory Sadler \\ ${ }^{1}$ Consultant Endocrine Surgeon, Departments of Endocrine Surgery, John Radcliffe Hospital, Oxford OX3 9DU, United Kingdom \\ ${ }^{2}$ Consultant Pathologist, Departments of Endocrine Surgery, John Radcliffe Hospital, Oxford OX3 9DU, United Kingdom \\ ${ }^{3}$ Consultant Cardiothoracic Surgeon, Departments of Cardiothoracic Surgery, John Radcliffe Hospital, Oxford OX3 9DU, United \\ Kingdom
}

${ }^{4}$ General Surgery (Eng), Department of Pathology, John Radcliffe Hospital, Oxford OX3 9DU, United Kingdom

Correspondence: Radu Mihai, Consultant Surgeon/Honorary Senior Clinical Lecturer, Department of Endocrine Surgery John Radcliffe Hospital, Headley Way, Headington, Oxford, OX3 9DU, United Kingdom, Fax: +44-1865-221189 e-mail: radumihai@doctors.org.uk

Metastases into the adrenal glands are increasingly diagnosed in patients with lung cancer, breast cancer, renal cell carcinoma, melanoma and lymphoma. The advent of laparoscopic adrenalectomy has triggered a growing interest in selecting patients with known malignancy and isolated adrenal metastases that could benefit from adrenalectomy. ${ }^{1}$ In this context, it is important to exclude synchronous functional adrenal tumors before proceeding to laparoscopic adrenal metastasectomy. For example, a recent case report described the rare possibility of simultaneous diagnosis of lung cancer and pheochromocytoma. ${ }^{2}$

We report a patient presenting with a small nonsmall cell lung cancer (NSCLC) and a pheochromocytoma who was found to have cancer-to-tumor metastases (i.e. pheochromocytoma infiltrated by metastatic lung cancer cells).

A 74 years old heavy smoker woman was found to have an abnormal chest $\mathrm{X}$-ray during investigations for polyarthralgia. A subsequent $\mathrm{CT}$ scan of her chest and abdomen described a $17 \mathrm{~mm}$ spiculated lesion in the left lower lobe of the lung, uncalcified, and associated with pleural tethering and with no evidence of mediastinal lymphadenopathy. In addition there was a $53 \mathrm{~mm}$ right adrenal mass. On direct questioning, the patient revealed episodes of sweats and heat intolerance and infrequent nocturnal palpitations over past 5 years. Blood pressure was normal (130/75 mm Hg). A fine needle lung biopsy demonstrated a non-small cell lung carcinoma. Lung function tests were normal: FEV1 of 2.2 (predicted normal 2.3) and FVC 3.0 (predicted normal 3.0), FEV1/FVC of 73\%. 24 hours urine samples showed raised metadrenaline $(36.62 \mu \mathrm{mol}$, normal range $0-1.4 \mu \mathrm{mol}$ ), normetadrenalines ( $8.7 \mu \mathrm{mol}$, normal range $<0.3 \mu \mathrm{mol}$ ) and 3-methoxytyramine (3.92 $\mu \mathrm{mol}$, normal range $0-2.75 \mu \mathrm{mol}$ ). An MIBG scan showed increased uptake in the right adrenal mass. Alpha blockade with Phenoxybenzamine $30 \mathrm{mg} /$ day was followed by beta blockade with Propranolol $50 \mathrm{mg} /$ day for 6 weeks before undergoing right transperitoneal laparoscopic adrenalectomy and left thoracotomy with left lower lobectomy. Postoperative recovery was uneventful.
On histopathological examination, the lung tumor was a moderately differentiated carcinoma with extensive lymphatic invasion and involvement of pleural surface. The peribronchial, paraesophageal and hilar nodes contained metastatic deposits (T2N2M1, stage 4). The adrenal gland measured $80 \times 40 \times 35$ $\mathrm{mm}$, weighed $75 \mathrm{gm}$ and represented a phaeochromocytoma extensively infiltrated by metastatic cells from the lung primary (Figs 1A and B). Chemotherapy was commenced three months after her operation. The patient died 10 months after the combined surgical procedure.

This report describes a patient with a synchronous diagnosis of pheochromocytoma and lung cancer, a combination that has only been described once previously. ${ }^{2}$ The unusual finding in our patient was that her adrenal tumor was invaded by metastatic lung cancer.

The American college of chest physicians guidelines suggest that resection of an isolated synchronous adrenal metastasis should be undertaken in selected patients with no other sites of metastases and a resectable (i.e. N0 or N1) primary nonsmall cell lung carcinoma (NSCLC). ${ }^{3}$ Several small series have reported improved survival in such patients (Table 1), with an overall 5-year survival of $10-23 \%$. In a recent series of 114 patients with NSCLC and adrenal metastases, those with synchronous metastases (42\%) were younger and had shorter survival when compared with those with metachronus metastases (mean 12 vs 32 months). ${ }^{4}$ These data fit well with the clinical outcome of the case presented.

Although the presence of two or more tumors in a single patient is not a rare occurrence, metastases into another tumor are rare. Less than 50 cases of cancer-to-cancer metastases have been identified in a recent publication. ${ }^{5}$ Generally the most frequent donor tumor is a lung cancer, and the most common recipient tumor is a renal cell carcinoma. Even less common are reports of metastases into a benign tumor. ${ }^{6} \mathrm{~T}$ our knowledge, this is the only case of metastasis into a pheochromocytoma from a lung cancer and adds to the two previous reports of 

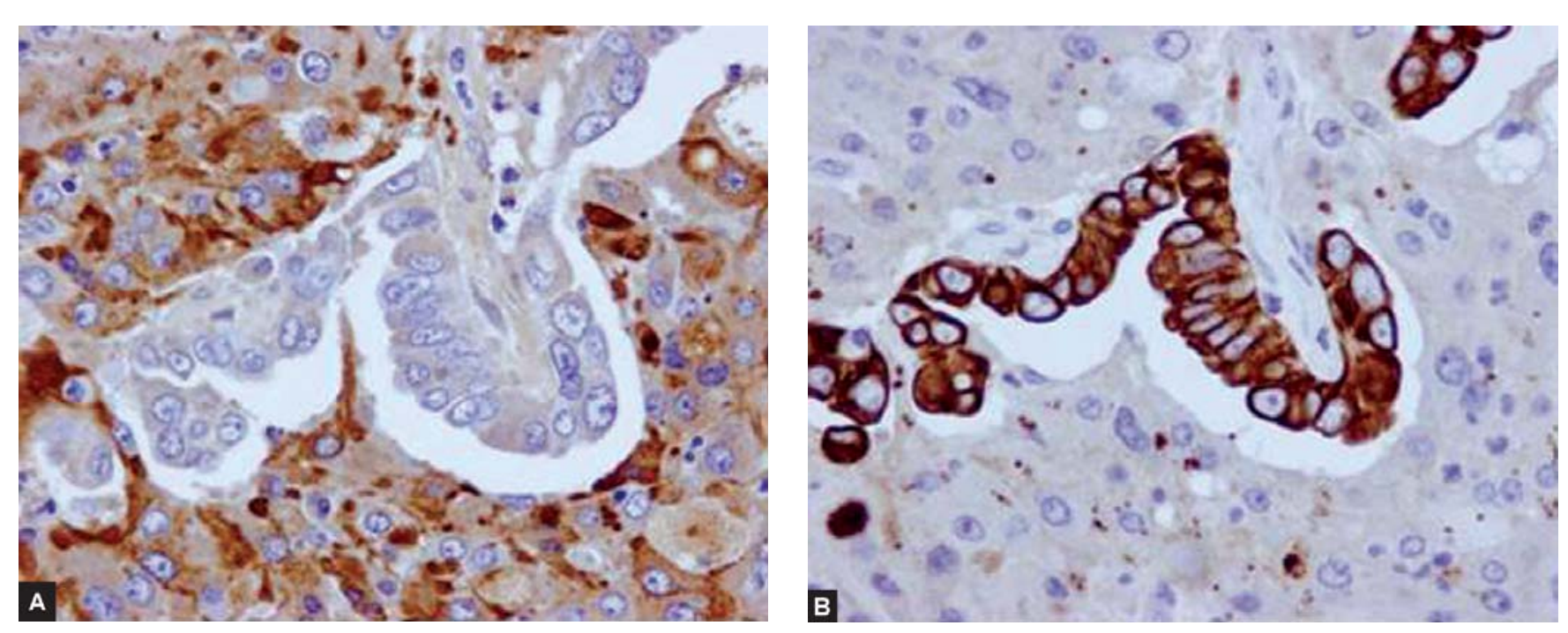

Figs 1A and B: Immunohistochemistry using primary antibodies against chromogranin $(A)$ and cytokeratin

(B) demonstrate infiltration of the neuroendocrine tumor with metastatic lung cancer cells ( $\times 400$ magnification)

metastasis from an ipsilateral renal cell carcinoma ${ }^{7}$ and breast cancer. $^{8}$

\section{REFERENCES}

1. Sturgeon C, Kebebew E. Laparoscopic adrenalectomy for malignancy. Surg Clin North Am. 2004;84(3):755-74.

2. Chen EP, Weber CJ, Smith CD, Miller JI Jr. Synchronous presentation of primary non-small cell lung carcinoma and pheochromocytoma. Ann Thorac Surg. 2002;74(3):924-26.

3. http://www.chestjournal.org/cgi/content/full/123/1_suppl/244S.

4. Tanvetyanon T, Robinson LA, Schell MJ, Strong VE, Kapoor R, Coit DG, Bepler G. Outcomes of adrenalectomy for isolated synchronous versus metachronous adrenal metastases in non- small-cell lung cancer: A systematic review and pooled analysis. J Clin Oncol. 2008 Mar 1;26(7):1142-47.

5. Moller MG, Gribbin T, Ebrom S, Padula G, Fitzgerald TL. Breast cancer metastatic to renal cell carcinoma. Surgery 2006;139:577-79.

6. Richardson J, Katamaya I. Neoplasm to neoplasm metastasis. Arch Path 1971;91:135-39.

7. Ozenç A, Ruacan S, Baykal A. Renal cell carcinoma and ipsilateral pheochromocytoma with neoplasm-to-neoplasm metastasis. J Urol. 1997;157(5):1831-32.

8. Seitz G, Schüder G. Neoplasm to neoplasm metastasis. Pheochromocytoma harboring a metastasis of breast cancer. Pathol Res Pract. 1987;182(2):228-32. 